\title{
Afiliação geográfica como possível preditor de comportamentos de multitarefa ${ }^{1}$
}

\author{
La afiliación geográfica como possible predictor del comportamiento \\ multitarefa
}

\section{Geographical affiliation as possible predictor of multitasking behaviours}

\author{
Cláudia Marina Mónica de Oliveira Barbosa ${ }^{2}$ \\ Luís Francisco Mendes Gabriel Pedro ${ }^{3}$
}

\begin{abstract}
Resumo
A multitarefa, especialmente combinada com o uso de diferentes tipos de meios de comunicação, tornou-se um comportamento comum na nossa vida quotidiana, sendo promovida por constantes desenvolvimentos tecnológicos em equipamentos de uso pessoal. Os comportamentos de multitarefa têm sido frequentemente estudados a partir de perspetivas individuais como a idade e o género. Há, contudo, menos estudos sobre como a origem, a afiliação cultural ou geográfica pode influenciar ou potenciar comportamentos de multitarefa. Este artigo tem como objectivo analisar uma possível ligação entre afiliação geográfica, uso de meios de comunicação e tendência para comportamentos multitarefa de um grupo de dez investigadores, todos do sexo masculino, todos com a mesma profissão e com o mesmo ambiente de trabalho, com diferentes afiliações geográficas, agrupados em diferentes grupos regionais (Europa, Norte de África, Ásia Meridional/Ocidental). Foram registadas diferenças no consumo de meios de comunicação, sendo os valores de consumo semanais do cluster europeu ( 76.3 horas) bastante abaixo da média, os do cluster norte-africano (104.83 horas) bastante acima da média e os do cluster asiático (83.17 horas) aproximados à média (86.92 horas/semana). Uma tendência semelhante verifica-se nos valores de multitarefa com vários meios de comunicação, apresentando o cluster europeu os valores mais baixos de comportamento multitarefa globalmente, mas também os valores mais baixos em relação a dez dos meios de comunicação. O cluster do Norte de África regista não só o maior índice de comportamento multitarefa dos três clusters, como valores parcelares de multitarefa mais elevados para oito dos meios de comunicação.
\end{abstract}

Palavras-Chave: afiliação geográfica; média tradicionais; multitarefa; novos média; consumo

\section{Resumen}

La multitarea, especialmente aplicada el uso de diferentes tipos de medios de comunicación, se ha convertido en un comportamiento común en nuestra vida cotidiana, siendo promovida por constantes desarrollos tecnológicos. Los comportamientos multitarea se han estudiado a menudo desde perspectivas individuales como la edad y el género. Sin embargo, hay menos estudios sobre cómo el origen geográfico, o la afiliación étnica y cultural pueden influenciar comportamientos de multitarea. Este artículo tiene como objetivo examinar una posible relación entre la afiliación geográfica, el uso de los medios de comunicación y la tendencia a realizar tareas múltiples de un grupo de 10 investigadores en una institución de investigación en Portugal, todos hombres, todos con la misma profesión y ambiente laboral, con diferentes afiliaciones geográficas y agrupados en diferentes

\footnotetext{
${ }^{1}$ Artigo apresentado durante o I Congresso Internacional Online de Estudos sobre Culturas - \#Culturas, realizado de forma online, em outubro de 2019.

2 Licenciada; Universidade de Aveiro, DIGIMEDIA, Dep. Comunicação e Arte; Aveiro, Portugal; cmmob@ua.pt
}

${ }^{3}$ Doutor; Universidade de Aveiro, DIGIMEDIA, Dep. Comunicação e Arte; Aveiro, Portugal; lpedro@ua.pt 
grupos regionales (Europa, Norte de África, Asia Meridional/Occidental). Se registraron diferencias en el consumo de varios medios de comunicación y en los valores de consumo totales, con los valores de consumo semanal del grupo europeo (76.3 horas) muy por debajo del promedio, los del grupo del norte de África (104.83 horas) muy por encima del promedio y los del grupo asiático (83.17 horas) aproximado al promedio (86.92 horas / semana). Una tendencia similar se registró para los valores de multitarea, con el clúster europeo con los valores más bajos para el comportamiento de multitarea a nivel global, pero también los valores más bajos para diez de los medios. El clúster del norte de África no solo tiene el índice de comportamiento multitarea más alto de los tres clústeres, sino que también tiene los valores de multitarea más altos para ocho de los medios.

Palabras claves: afiliación geográfica; medios tradicionales; multitarea; nuevos medios; consumo

\begin{abstract}
Multitasking, especially combined with the use of different types of media, has become a common behavior in our daily lives, promoted by constant technological developments in personal use equipment. Multitasking behaviors have frequently been studied from individual perspectives such as age and gender. There are, however, fewer studies on how the origin, cultural or geographical affiliation can influence or enhance multitasking behaviors. This article aims to analyze a possible link between geographical affiliation, media use and the tendency to multitask of a group of ten researchers, all male, all with the same profession and work environment, but with different geographical affiliations, grouped into different regional groups (Europe, North Africa, South / West Asia). Differences in media consumption were recorded, with the weekly consumption values of the European cluster (76.3 hours) well below average, those of the North African cluster (104.83 hours) well above average and those of the Asian cluster ( 83.17 hours) close to the average ( 86.92 hours/week). A similar trend is recorded for multimedia multitasking values, with the European cluster having the lowest values for multitasking behavior globally, but also the lowest values for ten of the media. The North African cluster not only has the highest multitasking behavior index of the three clusters, but also has the highest multitasking split values for eight of the media.
\end{abstract}

Keywords: geographical affiliation; traditional media; multitasking; new media; media use

\title{
1. Introdução
}

O conceito de "multitarefa" foi inicialmente investigado no âmbito da Psicologia Cognitiva, tendo a maior parte da investigação inicial tido como objetivo promover a eficiência nas organizações, dispondo os trabalhadores da melhor forma para atender às tarefas. A perspetiva de multitarefa como alternância de tarefas mentais foi introduzida por volta de 1920, tendo o desenvolvimento de meios de comunicação como rádio, televisão e mais tarde computador, originado o nascimento real da multitarefa segundo Hassoun (2012).

A American Psychology Association (APA) caracterizou a ocorrência de multitarefa nas situações em que alguém tenta realizar duas tarefas simultaneamente, alternar entre tarefas ou executar duas ou mais tarefas em rápida sucessão. Embora esta definição não especifique o tipo de tarefas executadas ou os meios usados para as realizar, é comum associar o conceito de multitarefa ao uso de um ou vários meios de comunicação, dando assim origem ao termo "media multitasking", ou seja, comportamentos de multitarefa com meios de comunicação.

A "afiliação geográfica" - geralmente pensada em termos de etnia, origem ou nacionalidade - é de díficil definição, sendo percebida e registada de forma diferente em diferentes países (Simon, 2011). Ao definir “identidade", Hogan (2009) refere-se à 
nacionalidade e afiliação geográfica (uma das inúmeras camadas da identidade de um indivíduo) como lealdade específica a países ou áreas geográficas, afirmando que a localização geográfica não se limita ao seu local de nascimento ou localização atual e em vez disso pode ser determinado pela sua mentalidade ou sistema de crenças. Para o seu estudo, Hogan (2009) seleciona "nacionalidade" quando se refere ao país geográfico em relação ao qual se sente a mais forte aliança ou lealdade. Acreditamos, porém, que esse termo possa causar confusão desnecessária neste estudo, já que metade dos participantes adquiriu a nacionalidade portuguesa. Optamos, portanto, por "afiliação geográfica", usada com significado semelhante também em Peters et al. (2014). Como todos os participantes têm afiliações diferentes, e para melhor comparar diferentes grupos regionais, optamos por agregá-los em clusters regionais baseados em proximidade geográfica, seguindo a hipótese postulada por Latané (1995) apud Harton \& Bourgeois (2004) de que ao longo do tempo, atitudes dentro de um grupo devem mostrar agrupamento regional; isto é, as pessoas estarão cada vez mais propensas a compartilhar atitudes semelhantes com as que vivem perto delas.

Vários aspetos têm sido avaliados como possíveis preditores de comportamentos de multitarefa com meios de comunicação, sendo os mais destacados o género e a idade. A afiliação geográfica como preditor de multitarefa tem recebido, até ao momento, pouca atenção na literatura, sendo os estudos mais relevantes os de Kononova et al. (2013, 2014, 2015), Voorveld et al. (2014) e Bowman et al. (2014).

A maioria destes estudos relaciona a dimensão geográfica com os conceitos de "monocronicidade" e "policronicidade", definidos pela primeira vez pelo antropólogo americano Edward T. Hall (1959) que definiu monocronicidade como a preferência por fazer uma atividade de cada vez. Hall argumentou que as culturas eram predominantemente monocrónicas ou policrónicas no que diz respeito à sua relação com o tempo. Com base no estudo de Hall, Morden (1999) propôs a seguinte uma escala demográfica que considera os seguintes grupos por ordem de progressão num continuum entre Monocronicidade e Policronicidade: Alemães; Suíços e Austríacos; Americanos; Escandinavos e Finlandeses; Britânicos, Canadianos e Neozelandeses; Australianos e Sul-Africanos; Japoneses; Holandeses e Belgas-Flamengos; Outras culturas americanas; Franceses e Belgas/Valões; Coreanos e Taiwaneses; Singapurenses; Checos, Eslovacos, Croatas e Húngaros; Chineses; Italianos do Norte; Chilenos; Outros Eslavos; Portugueses; Espanhóis, Italianos do Sul e povos mediterrânicos; Indianos e outros do sub continente indiano; Polinésios; Latino Americanos, Árabes e Africanos. 
Embora os conceitos de multitarefa e policronicidade sejam constructos teóricos distintos, eles são conceptualmente relacionáveis (Voorveld et al., 2014), sendo o primeiro o comportamento de realizar tarefas de forma simultânea e o segundo a preferência por esse tipo de comportamento. A maioria dos estudos mencionados questiona os respondentes em diferentes países e destaca diferenças económicas, políticas, culturais, e diferentes ecossistemas de comunicação. Este estudo difere dos referidos ao selecionar uma amostra semelhante a nível de: i) sexo, ii) idade, iii) qualificações, iv) ambiente de trabalho, v) área de residência; experienciando assim as mesmas características estruturais. Considerando que o principal fator diferenciador será a origem geográfica, tentaremos avaliar se é possível identificar tendências comuns à multitarefa entre indivíduos com afiliações geográficas semelhantes.

\section{Método}

Neste estudo foi utilizado o Media Use Questionnaire (MUQ) proposto por Ophir, Nass e Wagner (2009), que compreende questões sobre doze meios de comunicação: 1) média impressos, 2) televisão, 3) Youtube/TV online, 4) música, 5) áudio não musical, 6) jogos de computador, 7) chamadas de voz, 8) mensagens instantâneas, 9) SMS, 10) e-mail, 11) navegação Web e 12) outros aplicativos de computador. Os entrevistados devem indicar a) o total de horas semanais gastas com cada meio e b) com que frequência usam diferentes meios concorrentes para cada meio primário; as respostas a estas perguntas permitem aferir o Media Multitasking Index (MMI) de cada participante, calculando uma soma entre o uso do meio primário ponderado pela percentagem de tempo gasto com cada secundário.

O questionário foi administrado em inglês, em formato papel e lápis e foi apresentado a 10 participantes de 10 países diferentes: Reino Unido [UK], Espanha [SP], Grécia [GR], Portugal [PT], Egipto [EG], Sudão [SD], Líbia [LY], Irão [IR], Iraque [IQ], e Paquistão [PK]). Todos os participantes são do sexo masculino, doutorados em Engenharia, a trabalhar no mesmo instituto, com um perfil educacional e profissional comum. A idade média do grupo é de 39,6 anos [M=39.6; $\mathrm{SD}=5.94]$, tendo o respondente mais jovem 32 e o mais velho 50 anos. Dada a sua referência geográfica, os participantes foram agrupados em três clusters regionais: C1/Europa [UK, SP, GR, PT], C2/Norte de África [EG, LY, SD] e C3/Ásia Meridional/Ocidental [IQ, IQ, PK], sendo os clusters regionais e as afiliações geográficas apresentados na figura 1 . 


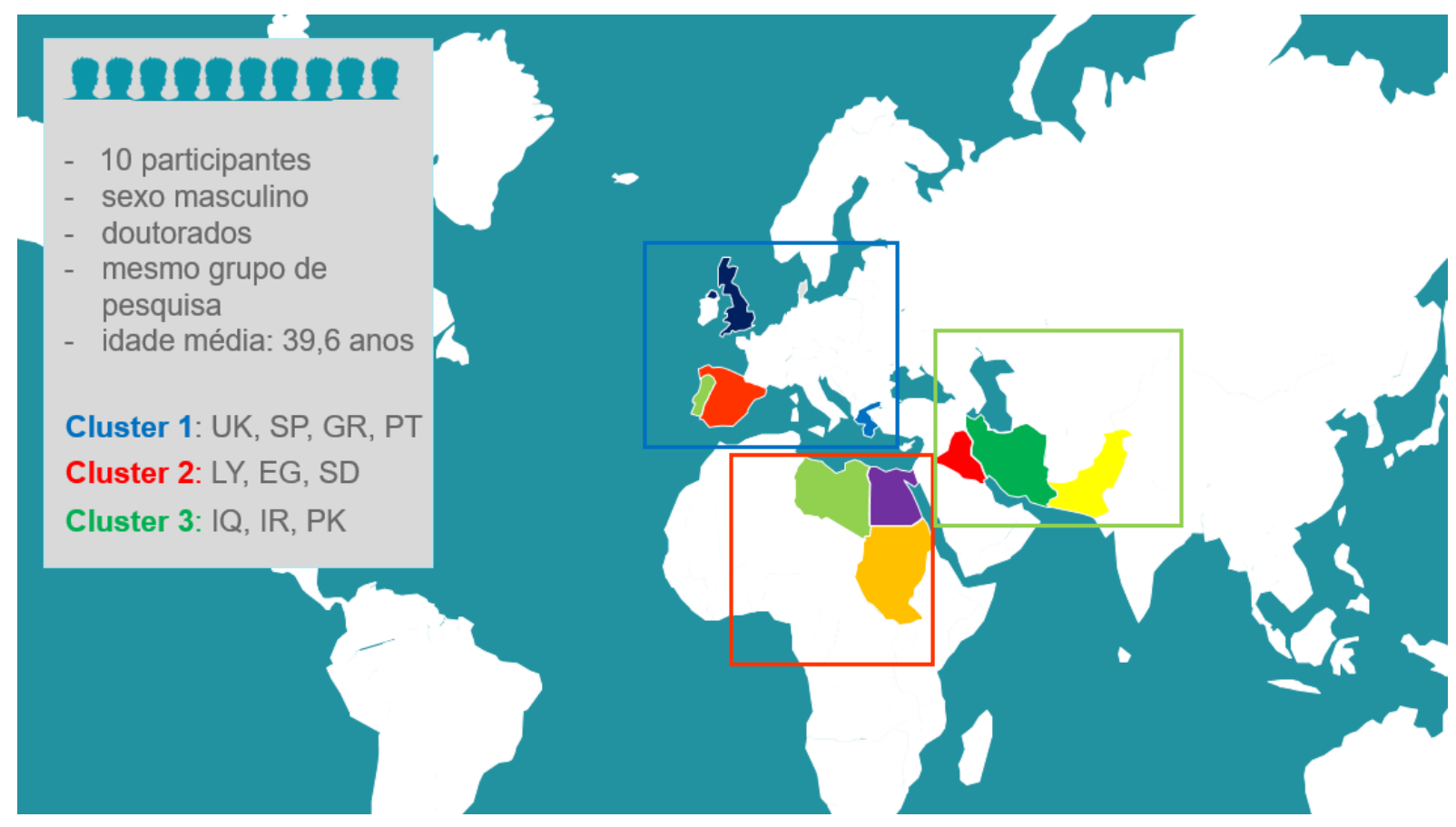

Figura 1 - Participantes no estudo

Fonte: autoria própria

\section{Resultados}

O questionário permite-nos obter os seguintes resultados: o número de horas que cada participante dedica ao uso de diferentes meios de comunicação e as utilizações de meios de comunicação em simultâneo com o uso de cada meio primário (expresso através do Media Multitasking Index).

A tabela 1 apresenta as horas semanais de uso indicadas pelos participantes em relação a cada um dos doze meios de comunicação analisados, apresentados individualmente, assim como a média global de uso de cada meio de comunicação por semana.

Tabela 1 - Uso de meios de comunicação: número de horas reportados por semana, por meio

\begin{tabular}{|c|c|c|c|c|c|c|c|c|c|c|c|}
\hline $\begin{array}{l}\text { Horas de uso de meios de } \\
\text { comunicação como meio de } \\
\text { comunicação primário }\end{array}$ & $\begin{array}{c}1 \\
\text { UK }\end{array}$ & $\begin{array}{c}2 \\
\text { SP }\end{array}$ & $\begin{array}{c}3 \\
\text { GR }\end{array}$ & $\begin{array}{c}4 \\
\mathrm{PT}\end{array}$ & $\begin{array}{c}5 \\
\mathrm{EG}\end{array}$ & $\begin{array}{c}6 \\
\text { LY }\end{array}$ & $\begin{array}{c}7 \\
\text { SD }\end{array}$ & $\begin{array}{c}8 \\
\mathrm{PK}\end{array}$ & $\begin{array}{c}9 \\
\text { IR }\end{array}$ & $\begin{array}{l}10 \\
\text { IQ }\end{array}$ & Média \\
\hline Média impressos & 1 & 10 & 18 & 0,1 & 10 & 5 & 16 & 0 & 10 & 1 & 7,11 \\
\hline Televisão & 7 & 1 & 1 & 6 & 24 & 21 & 2,5 & 2 & 10 & 14 & 8,85 \\
\hline Youtube / TV online & 1 & 8 & 15 & 1 & 10 & 7 & 2 & 14 & 7 & 3 & 6,8 \\
\hline Música & 0 & 1 & 4 & 3 & 28 & 4 & 2 & 0 & 2 & 14 & 5,8 \\
\hline
\end{tabular}


RELACult - Revista Latino-Americana de Estudos em Cultura e Sociedade

Revista Latinoamericana de Estudios en Cultura y Sociedad | Latin American Journal of Studies in Culture and Society V. 06, ed. especial, mar., 2020, artigo n ${ }^{\circ} 1791$ | claec.org/relacult | e-ISSN: 2525-7870

\begin{tabular}{|c|c|c|c|c|c|c|c|c|c|c|c|}
\hline Áudio não-musical & 0 & 4 & 0 & 0,5 & 0 & 1 & 2 & 0 & 1 & 2 & 1,05 \\
\hline Jogos de computador & 0 & 10 & 0 & 0 & 0 & 1 & 4 & 0 & 0 & 0 & 1,5 \\
\hline Chamadas de voz & 3 & 0,5 & 1 & 0,5 & 1 & 5 & 2 & 3 & 1 & 1 & 1,8 \\
\hline Mensagens instantâneas & 1 & 3 & 0,5 & 0,5 & 20 & 1 & 1 & 0 & 0,5 & 1 & 2,85 \\
\hline SMS & 0,5 & 0 & 0,1 & 0,5 & 1 & 1 & 1 & 1 & 0,5 & 0,5 & 0,61 \\
\hline Email & 14 & 5 & 70 & 0,5 & 15 & 24 & 15 & 20 & 8 & 14 & 18,55 \\
\hline Navegação web Totais & 51,5 & 82,5 & 149,6 & 21,6 & 137 & 90 & 87,5 & 85 & 80 & 84,5 & 86,92 \\
\hline
\end{tabular}

Fonte: autoria própria

Verifica-se uma grande variação de número de horas semanais reportadas em relação ao uso de meios de comunicação, sendo o participante que declara menos horas de uso, o português, e o que declara o maior número de horas de uso, o participante de origem grega. Em relação aos meios de comunicação em si, os meios menos utilizados por todos os participantes são o SMS e o áudio não musical, sendo os meios mais utilizados o email e outros aplicativos de computador.

A figura 2 apresenta o número de horas semanais de uso de meios de comunicação por clusters regionais e por elementos individuais de cada cluster regional.

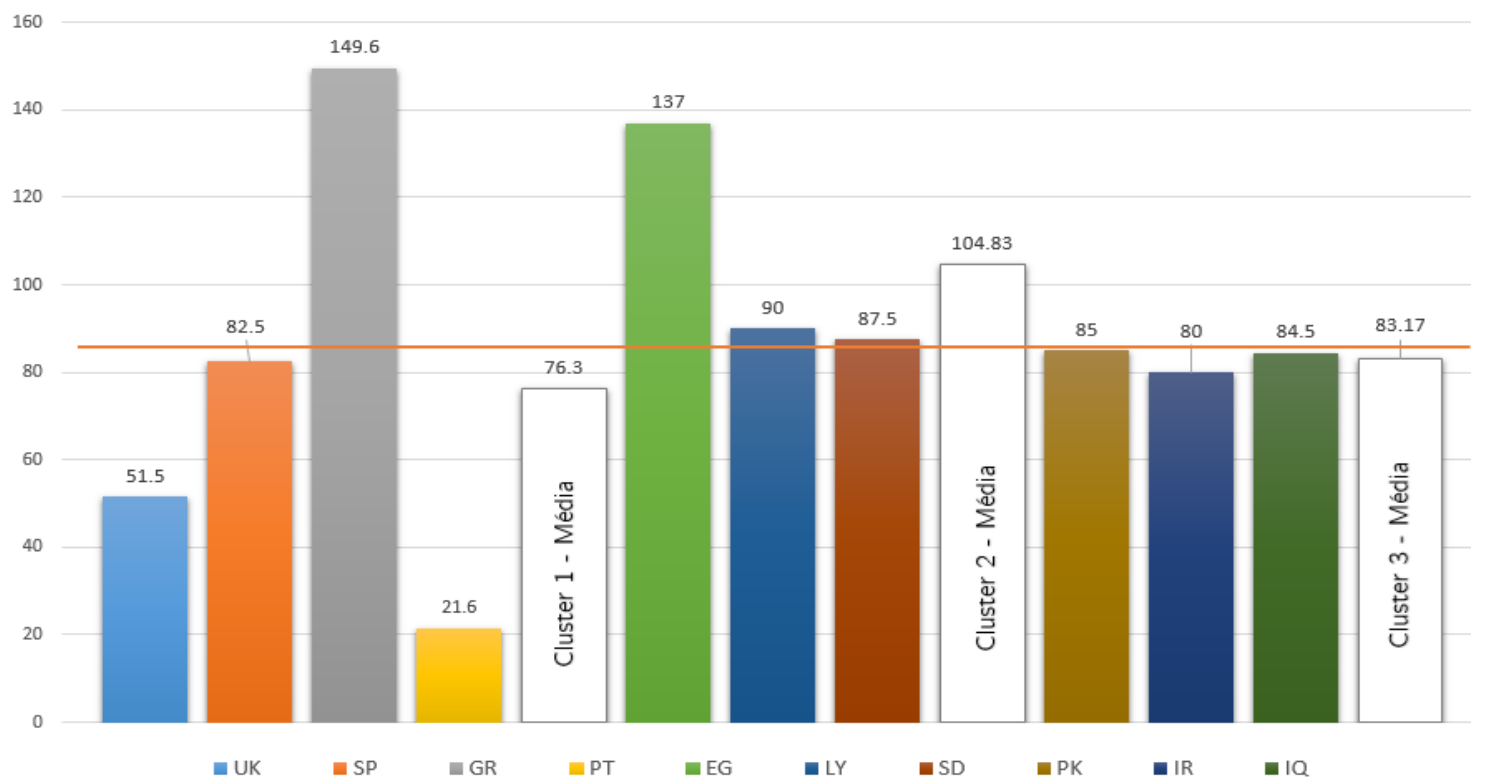

Figura 2 - Uso de meios de comunicação por participante e por cluster geográfico (horas/semana).

Fonte: autoria própria 
RELACult - Revista Latino-Americana de Estudos em Cultura e Sociedade

Revista Latinoamericana de Estudios en Cultura y Sociedad | Latin American Journal of Studies in Culture and Society V. 06, ed. especial, mar., 2020, artigo n ${ }^{\circ} 1791$ | claec.org/relacult | e-ISSN: 2525-7870

Verifica-se que o valor do cluster 1/Europa se situa bastante abaixo da média (76.3 horas), o valor do cluster 2/Norte de África bastante acima da média (104.83 horas) sendo o valor do cluster 3/Ásia (83.17 horas) o mais aproximado da média global (86.92 horas/semana, ou seja sensivelmente metade das horas existentes numa semana).

Tabela 2 - Media Multitasking Index (índice de comportamentos de multitarefa), por participante, por meio primário

\begin{tabular}{|c|c|c|c|c|c|c|c|c|c|c|c|c|c|c|}
\hline $\begin{array}{c}\text { Meios de } \\
\text { comunicação } \\
\text { primários }\end{array}$ & $\begin{array}{c}1 \\
\mathrm{UK}\end{array}$ & $\begin{array}{c}2 \\
\text { SP }\end{array}$ & $\begin{array}{c}3 \\
\text { GR }\end{array}$ & $\begin{array}{c}4 \\
\text { PT }\end{array}$ & $\mathrm{C} 1$ & $\begin{array}{c}5 \\
\mathrm{EG}\end{array}$ & $\begin{array}{c}6 \\
\text { LY }\end{array}$ & $\begin{array}{c}7 \\
\text { SD }\end{array}$ & $\mathrm{C} 2$ & $\begin{array}{c}8 \\
\text { PK }\end{array}$ & $\begin{array}{c}9 \\
\text { IR }\end{array}$ & $\begin{array}{l}10 \\
\text { IQ }\end{array}$ & $\mathrm{C} 3$ & Méd. \\
\hline $\begin{array}{c}\text { Média } \\
\text { impressos }\end{array}$ & 0,006 & 0,081 & 0,360 & 0,003 & 0,113 & 0,171 & 0,296 & 1,280 & 0,582 & 0,000 & 0,374 & 0,035 & 0,136 & 0,261 \\
\hline Televisão & 0,090 & 0,000 & 0,018 & 0,917 & 0,256 & 0,699 & 0,777 & 0,076 & 0,517 & 0,055 & 0,541 & 0,717 & 0,438 & 0,389 \\
\hline $\begin{array}{c}\text { Youtube / TV } \\
\text { online }\end{array}$ & 0,000 & 0,000 & 0,267 & 0,122 & 0,097 & 0,243 & 0,180 & 0,076 & 0,166 & 0,441 & 0,438 & 0,178 & 0,352 & 0,194 \\
\hline Música & 0,000 & 0,008 & 0,098 & 0,413 & 0,130 & 0,887 & 0,132 & 0,076 & 0,365 & 0,000 & 0,108 & 0,717 & 0,275 & 0,244 \\
\hline $\begin{array}{l}\text { Áudio não- } \\
\text { musical }\end{array}$ & 0,000 & 0,000 & 0,000 & 0,008 & 0,002 & 0,000 & 0,026 & 0,060 & 0,029 & 0,000 & 0,054 & 0,103 & 0,052 & 0,025 \\
\hline $\begin{array}{c}\text { Jogos de } \\
\text { computador }\end{array}$ & 0,000 & 0,161 & 0,000 & 0,000 & 0,040 & 0,000 & 0,018 & 0,122 & 0,047 & 0,000 & 0,000 & 0,000 & 0,000 & 0,030 \\
\hline $\begin{array}{c}\text { Chamadas de } \\
\text { voz }\end{array}$ & 0,039 & 0,004 & 0,020 & 0,023 & 0,021 & 0,022 & 0,092 & 0,107 & 0,073 & 0,095 & 0,017 & 0,016 & 0,042 & 0,043 \\
\hline $\begin{array}{l}\text { Mensagens } \\
\text { instantâneas }\end{array}$ & 0,013 & 0,036 & 0,014 & 0,023 & 0,022 & 0,435 & 0,022 & 0,015 & 0,157 & 0,000 & 0,031 & 0,059 & 0,030 & 0,065 \\
\hline SMS & 0,000 & 0,000 & 0,000 & 0,000 & 0,000 & 0,000 & 0,000 & 0,000 & 0,000 & 0,000 & 0,000 & 0,000 & 0,000 & 0,000 \\
\hline Email & 0,000 & 0,041 & 1,549 & 0,023 & 0,403 & 0,511 & 1,149 & 0,398 & 0,686 & 0,473 & 0,398 & 0,659 & 0,510 & 0,520 \\
\hline $\begin{array}{c}\text { Navegação } \\
\text { web }\end{array}$ & 0,221 & 0,061 & 0,154 & 0,367 & 0,201 & 0,442 & 0,294 & 0,283 & 0,340 & 0,473 & 1,000 & 0,828 & 0,767 & 0,412 \\
\hline \multirow{2}{*}{$\begin{array}{l}\text { Outros } \\
\text { aplicativos de } \\
\text { computador }\end{array}$} & 0,045 & 0,708 & 0,529 & 0,458 & 0,435 & 0,546 & 0,443 & 0,471 & 0,487 & 0,591 & 0,999 & 0,788 & 0,793 & 0,558 \\
\hline & 0,414 & 1,100 & 3,009 & 2,356 & 1,720 & 3,956 & 3,430 & 2,963 & 3,450 & 2,128 & 3,960 & 4,101 & 3,396 & 2,742 \\
\hline
\end{tabular}

Fonte: autoria própria

A figura 3 mostra o Media Multitasking Index (MMI) total para cada um dos participantes, bem como o MMI médio para cada um dos clusters geográficos. 
RELACult - Revista Latino-Americana de Estudos em Cultura e Sociedade

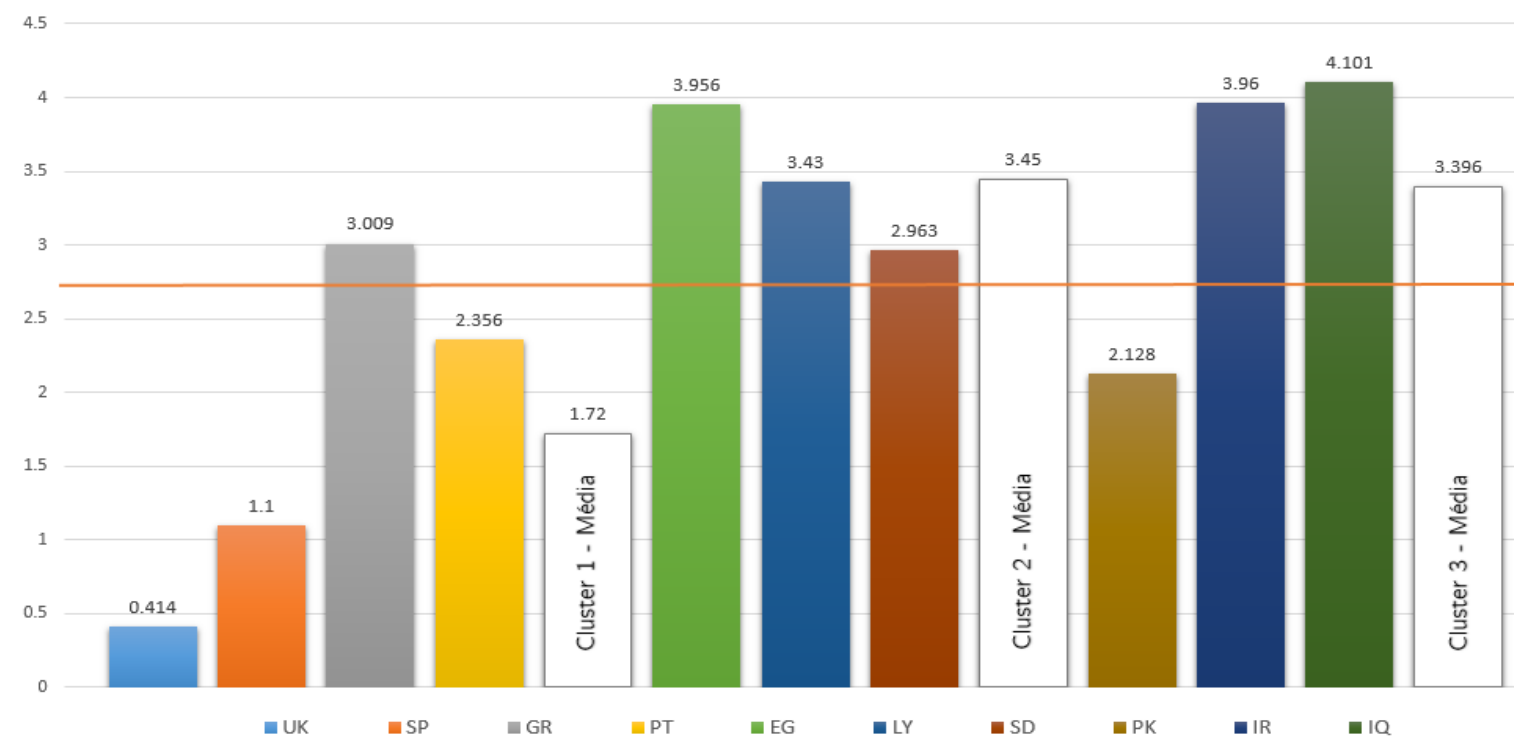

Figura 3 - Comportamentos multitarefa por participante e por cluster geográfico.

Fonte: autoria própria

Os MMIs dos 10 participantes variam de 0,414 (hábitos de multitarefa muito baixos) a 4,101 (hábitos de multitarefa muito altos), sendo a média de todos os participantes 2,742.

\section{Discussão}

No que diz respeito às horas de uso de meios de comunicação, o cluster 2/Norte de África regista valores bastante acima da média, sendo que todos os seus participantes reportam valores altos. No entanto o valor mais elevado registado é o do participante 3 [GR] que se destaca dos restantes participantes do Cluster 1/Europa, todos com valores relativamente baixos. O grupo mais homogéneo é o Cluster 3/Ásia, com valores de uso bastante similares. Os meios mais usados por cluster são: Cluster 1 (email; aplicações de computador e meios impressos); Cluster 2 (email; aplicações de computador e televisão); Cluster 3 (aplicações de computador; navegação na internet e email); sendo os menos usados: Cluster 1 (SMS); Cluster 2 (áudio não musical e SMS); Cluster 3 (jogos de computador).

Uma breve análise dos resultados dos três clusters revela que o MMI calculado para o Cluster 1/Europa (MMI=1.7) está abaixo da média global registada (MMI=2.7). Este cluster regista novamente uma grande discrepância nas respostas individuais, embora seja de referir não só que 3 dos 4 participantes do cluster 1 estão abaixo da média global, mas que estes representam também 3 dos 4 resultados mais baixos, suportando o argumento de que a maioria dos membros do Cluster 1 não só passa menos horas a interagir com diferentes meios como também tende a usá-los menos simultaneamente. O resultado geral do Cluster 2/Norte de África está, novamente, acima da média, devido ao fato de todos os membros do Cluster 2 
fazerem parte dos participantes com os mais altos índices multitarefa. Será interessante referir que os resultados do cluster 2 representam o dobro do índice global do cluster 1 . O cluster 3 regista o valor mais próximo da média, e um índice geral que é apenas 0,05 pontos inferior ao do Cluster 2, para um total de 20 horas de uso a menos. Embora não tão ativos em multitarefa como os membros do Cluster 2, os participantes do Cluster 3 estão definitivamente num patamar de comportamentos multitarefa acima dos membros do Cluster 1.

Comparações entre indivíduos de países tradicionalmente caracterizados como monocrónicos e indivíduos de áreas definidas como policrónicas não foram possíveis, uma vez que, seguindo a Escala Demográfica de Morden, apenas um dos entrevistados [UK, que curiosamente apresenta - de forma bastante clara - o valor de MMI mais baixo] foi considerado como pertencente a uma cultura tradicionalmente monocrónica.

\section{Conclusões}

Um dos grandes constrangimentos deste estudo foi a amostra limitada: uma vez que todos os participantes são originários de diferentes países, para tentar avaliar de que forma a distribuição geográfica afecta a tendência para a multitarefa, foram agrupados em clusters regionais, que podem incluir países que, embora geograficamente próximos possam exibir diferenças culturais significativas. A participação de mais respondentes com a mesma afiliação geográfica seria necessária para validar completamente algumas das suposições. Acreditamos, no entanto, que há dados suficientes neste estudo para lançar a possibilidade da existência de uma tendência para grupos geograficamente similares usarem meios de comunicação - de forma individual ou simultânea - de um modo semelhante.

\section{Referências}

AMERICAN PSYCHOLOGICAL ASSOCIATION. Multitasking: Switching costs. Disponível em: http://www.apa.org/research/action/multitask.aspx (Link em Site).

HALL. T. The silent language. N.Y: Doubleday, 1959 (Obra Completa).

HASSOUN, D. Costly attentions: Governing the media multitasker. Continuum. [s.1], v.26, p. 653-664, 2012. Disponível em: https://doi.org/10.1080/10304312.2012.698041. Acesso em 02 dez. 2016. (Artigo em Periódico Digital)

HARTON, H. C.; BOURGEOIS, M.J. Cultural Elements Emerge From Dynamic Social Impact. In: SCHALLER, M.; CRANDALL, C.S. (Org.). The psychological foundations of culture. Mahwah, NJ, US: Lawrence Erlbaum Associates Publishers, 2004. p. 41-75. (Capítulo de Livro) 
HOGAN, S. Globalization's Impact on Identity Through Billboard Advertisements: Independent Study Project (ISP) Collection, 654. 2009. Disponível em: http://digitalcollections.sit.edu/isp_collection/654 . (Relatório de trabalho).

KONONOVA, A. Multitasking Across Borders: A Cross-National Study of Media Multitasking Behaviors, Its Antecedents, and Outcomes. International Journal of Communication, 7(23): 1-20. (Artigo em Periódico Digital)

KONONOVA, A.; ZASORINA, T.; DIVEEVA, N.; KOKOEVA, A.; CHELOKYAN, A. Multitasking Goes Global: Multitasking With Traditional And New Electronic Media And Attention To Media Messages. International Communication Gazette, [s.1], v.76, n. 8, p.617-640, 2014. Disponível em: https://doi.org/10.1177/1748048514548533. Acesso em 20 dez. 2017. (Artigo em Periódico Digital)

KONONOVA, A.; CHIANG, Y.-H. Why Do We Multitask With Media? Predictors of Media Multitasking Among Internet Users In The United States And Taiwan. Computers In Human Behavior, [s.1], v.50, p.31-41. 2015 Disponível em: https://doi.org/10.1016 /j.chb.2015.03.052. Acesso em 20 dez. 2017. (Artigo em Periódico Digital)

MORDEN, T. Models Of National Culture - A Management Review. Cross Cultural Managemen. [s.1], v.1, p.19-44, 1999. Disponível em: https://doi.org/10.1108/ 13527609910796915. Acesso em 02 fev. 2018. (Artigo em Periódico Digital)

OPHIR E.; NASS, C.; WAGNER, A. D., Cognitive Control In Media Multitaskers. Proceedings of National Academy Of Sciences. [s.1], v.37, p.15583-15587, 2009. Disponível em: https://doi. org/10.1073/pnas.0903620106. Acesso em 02 dez. 2016. (Artigo em Periódico Digital)

PETERS, K.; DANIELS, K.; HODGKINSON, G.P.; HASLAM, S.A., Experts' judgments of management journal quality: an identity concerns model. Journal of Management, 40 (7). pp. 1785-1812, 1999. Disponível em doi: https://doi.org/10.1177/0149206311434532 Acesso em 02 jun. 2019 (Artigo em Periódico Digital)

SIMON, P. Collecting ethnic statistics in Europe: a review. Ethnic and Racial Studies, 35, 8, 2011. Disponível em: https://doi.org/10.1080/01419870.2011.607507 (Artigo em Periódico Digital)

VOORVELD, H.; SEGJIN, C.; KETELAAR, P.; SMIT, E. Investigating the Prevalence and Predictors of Media Multitasking across Countries. International Journal Of Communication, 8, 23, 2014. Disponível em: https://ijoc.org/index.php/ijoc/article /view/2556 (Artigo em Periódico Digital) 